\title{
Counting by weighing: know your numbers with confidence
}

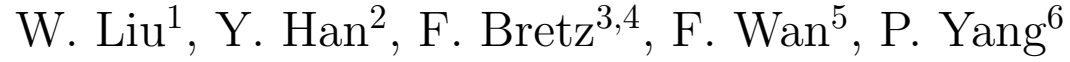 \\ ${ }^{1}$ S3RI and School of Mathematics \\ University of Southampton, SO17 1BJ, UK \\ ${ }^{2}$ University of Exeter Medical School, Exeter University, UK \\ ${ }^{3}$ Novartis Pharma AG, Basel, 4002, Switzerland \\ ${ }^{4}$ Shanghai University of Finance and Economics \\ People's Republic of China \\ ${ }^{5}$ Department of Mathematics and Statistics, Lancaster University, UK \\ ${ }^{6}$ Department of Statistics \\ The Chinese University of Hong Kong, Hong Kong
}

December 2015 


\begin{abstract}
Counting by weighing is often more efficient than counting manually which is time consuming and prone to human errors, especially when the number of items (e.g. plant seeds, printed labels, coins) is large. The published papers in the statistical literature have focused on how to count, by weighing, a random number of items that is close to a pre-specified number in some sense. This paper considers the new problem, arising from a consultation with a company, of making inference about the number of one-penny coins in a bag with known weight for infinitely many bags, by using the estimated distribution of coin weight from one calibration data set only. Specifically, a lower confidence bound has been constructed on the number of one-penny coins for each of infinitely many future bags of one-penny coins, as required by the company. As the same calibration data set is used repeatedly in the construction of all these lower confidence bounds, the interpretation of coverage frequency of the lower confidence bounds proposed in this paper is different from that of a usual confidence set.
\end{abstract}

Keywords: Confidence bound; Confidence level; Confidence set; Coverage frequency; Statistical inference. 


\section{Introduction}

Clients bring in bags of one-penny coins to exchange for bank notes or one- or two-pound coins. The company needs to know the numbers of coins in the bags in order to pay the clients. To count the number of coins in a bag manually is time consuming and prone to human errors, especially when the number is large. Counting by weighing offers an efficient alternative. The company can weigh the bags and use the (net) weight $W_{i}$ grams of a bag to infer the number $n_{i}$ of coins in the bag for infinitely many future bags $i=1,2, \cdots$. Nowadays weighing scales to the accuracy of one hundredth gram (g) are commonly available, and electronic scales with sensitivity of 0.01 milligram are not uncommon. Hence it is assumed throughout this paper that the weight of the coins in a bag can be weighed accurately.

If all the one-penny coins weigh exactly $3.56 \mathrm{~g}$ as specified by the Royal Mint, then there are exactly $W_{i} / 3.56$ coins in a bag with weight $W_{i}$. However, due to production variation and wear and tear and different types or amounts of foreign materials stuck to the coins after being in circulation, the weights of the one-penny coins cannot be exactly $3.56 \mathrm{~g}$ and can be assumed to follow a certain probability distribution. It is assumed throughout this paper, as most papers on counting by weighing, that the weight of a one-penny coin $U$ has a normal distribution $N\left(\mu, \sigma^{2}\right)$ for some $\mu$ and $\sigma^{2}$, which is sensible from the information we have on coin weights.

Due to the randomness in $U$, the exact number of one-penny coins in a bag with weight $W_{i}$ can no longer be pin-pointed. To make inference about the $n_{i}$ 's, information about the unknown $\mu$ and $\sigma^{2}$ is clearly required. For this, the company carries out the following calibration experiment to estimate $\mu$ and $\sigma$ : weigh the $j$ th bag known to contain $m_{j}$ one-penny coins and record the weight $W_{0 j}, j=1, \cdots, k$. Assume $k \geq 2$ in order to be able to estimate $\sigma^{2}$. This includes the special case that each bag contains only one one-penny coin and so 
$W_{0 j}, j=1, \cdots, k$ are the weights of a random sample of $k$ one-penny coins. The calibration data $\mathcal{E}=\left\{\left(m_{j}, W_{0 j}\right): j=1, \cdots, k\right\}$ is then used to make inference about $n_{i}$ 's for infinitely many future bags $i=1,2, \cdots$. Specifically, a lower confidence bound on the exact number $n_{i}$ of one-penny coins in a bag with weight $W_{i}$ is sought, which provides more useful information than a point estimate of $n_{i}$, for $i=1,2, \cdots$.

In this paper, a set of lower confidence bounds $L\left(W_{i}\right)$ on $n_{i}$ using the weight $W_{i}(i=1,2, \cdots)$ is provided. Note that these lower confidence bounds are constructed using the same calibration data $\mathcal{E}$ but different future weights $W_{i}$ and so treat the two sources of randomness in $\mathcal{E}$ and in a future weight $W_{i}$ differently. As a result, the coverage frequency interpretation of the set of confidence bounds proposed in this paper is different from that of a usual confidence set.

Several statistical papers have discussed counting by weighing (Nelson, 1983, Guttman and Menzefricke, 1986, Yu, 1989, Mullennix, 1990, Nickerson, 1993). The focus of these papers is on how to count (by weighing) a random number $\hat{n}$ of items, e.g. plant seeds, bolts or printed labels, that is close to a pre-specified number $n$ in a certain sense. This is different from the problem addressed in this paper in which the number $n_{i}$ is a non-random unknown parameter and a lower confidence bound on $n_{i}$ is required. The papers Ridout and Suntheralingam (1997), Ridout and Roberts (1997) and Nickerson (2003, 2008) consider the effect of using $\hat{n}$ instead of $n$ on some seed testing statistical procedures. Arntzen et al. (1994) uses the weight to point-estimate the number of eggs in a cyst of nematodes by fitting a linear regression model of the number of eggs on the weight. It is interesting to note that counting the number of eggs manually needs to crush the cysts and so is destructive, while counting by weighing offers a non-destructive alternative.

The layout of the paper is as follows. The simple situation where $\mu$ and $\sigma^{2}$ are assumed to be known is considered in Section 2 to illustrate the construction method, and to motivate 
the form of confidence sets in Section 3. The more realistic situation where both $\mu$ and $\sigma^{2}$ are unknown parameters is studied in Section 3. An example is given in Section 4. Finally Section 5 contains conclusions and discussion.

\section{Known $\mu$ and $\sigma^{2}$}

In this section, the values of $\mu$ and $\sigma^{2}$ are assumed to be known, which helps to motivate and understand the construction method and form of the confidence sets developed in Section 3 for the more realistic situation that both $\mu$ and $\sigma^{2}$ are unknown. Note the distribution $\left(W_{i}-n_{i} \mu\right) / \sqrt{n_{i} \sigma^{2}} \sim N(0,1)$. We construct the following confidence set for $n_{i}$ by using Neyman's (1937) method of inverting a family of acceptance sets for testing $H_{0}: n_{i}=n$ against $H_{a}: n_{i}>n$ for each natural number $n$ :

$$
\mathcal{C}\left(W_{i}\right)=\left\{n:\left(W_{i}-n \mu\right) / \sqrt{n \sigma^{2}} \leq c\right\}
$$

where $c$ is a suitably chosen critical constant whose determination is discussed next.

As pointed out in the introduction, it is desirable that, among the infinitely many confidence sets $\mathcal{C}\left(W_{i}\right)$ for possibly different parameters $n_{i}(i=1,2, \cdots)$, at least proportion $\beta$ will contain the true $n_{i}$ for the pre-specified $\beta$ (close to one), that is,

$$
\liminf _{N \rightarrow \infty} \frac{1}{N} \sum_{i=1}^{N} I_{\left\{n_{i} \in \mathcal{C}\left(W_{i}\right)\right\}} \geq \beta
$$

where $I_{A}$ denotes the indicator function of set $A$ and so $\frac{1}{N} \sum_{i=1}^{N} I_{\left\{n_{i} \in \mathcal{C}\left(W_{i}\right)\right\}}$ is the proportion of the confidence sets $\mathcal{C}\left(W_{i}\right)$ that contain the true $n_{i}$. Note that

$$
\lim _{N \rightarrow \infty} \frac{1}{N} \sum_{i=1}^{N} I_{\left\{n_{i} \in \mathcal{C}\left(W_{i}\right)\right\}}=\lim _{N \rightarrow \infty} \frac{1}{N} \sum_{i=1}^{N} P\left\{n_{i} \in \mathcal{C}\left(W_{i}\right)\right\}=\lim _{N \rightarrow \infty} \frac{1}{N} \sum_{i=1}^{N} \Phi(c)=\Phi(c),
$$

where the first equality above follows from the classical strong law of large numbers (cf. Chow and Teicher, 1978, Page 333), and the second from the definition of $\mathcal{C}\left(W_{i}\right)$ in (1). Hence we 
set $c=z_{\beta}$, the $\beta$-quantile of the $N(0,1)$ distribution, which guarantees the property in $(2)$ with equality.

The interpretation of the property in (2) is similar to coverage frequency of a standard confidence set. Property (2) is not surprising since $\mathcal{C}\left(W_{i}\right)$ is a standard $\beta$ level confidence set for $n_{i}$ for each $i$, even though the confidence sets $\mathcal{C}\left(W_{i}\right)$ are for possibly different parameters $n_{i}(i=1,2, \cdots)$. The situation for the unknown $\mu$ and $\sigma^{2}$ in Section 3 is different, however, due to that the same calibration data $\mathcal{E}$ is used repeatedly in the construction of all the infinitely many $\mathcal{C}\left(W_{i}\right)^{\prime}$ s.

It is straightforward to show that the confidence set $\mathcal{C}\left(W_{i}\right)$ in $(1)$ is given by the lower confidence bound

$$
L\left(W_{i}\right)=\frac{2 \mu W_{i}+c^{2} \sigma^{2}-c \sigma \sqrt{c^{2} \sigma^{2}+4 \mu W_{i}}}{2 \mu^{2}}
$$

or, equivalently, all the natural numbers contained in the half interval $\left[L\left(W_{i}\right),+\infty\right)$.

Note that a sensible point estimator of $n_{i}$ is $W_{i} / \mu$. Hence the distance between the lower confidence bound $L\left(W_{i}\right)$ and the point estimator $W_{i} / \mu$ of $n_{i}$ is $c \sigma\left(\sqrt{c^{2} \sigma^{2}+4 \mu W_{i}}-c \sigma\right) /\left(2 \mu^{2}\right)$, which is an increasing function of $\sigma$ and $\beta$ as expected.

\section{Unknown $\mu$ and $\sigma^{2}$}

Now we consider the more realistic situation where both $\mu$ and $\sigma^{2}$ are unknown and so need to be estimated from the calibration data $\mathcal{E}$ independently of the future weights $W_{i}$ 's.

The calibration data $\mathcal{E}=\left\{\left(m_{j}, W_{0 j}\right): j=1, \cdots, k\right\}$ can be used to estimate $\mu$ and $\sigma^{2}$ in the following way. Note that $Y_{i}:=W_{i} / \sqrt{n_{i}} \sim N\left(\sqrt{n_{i}} \mu, \sigma^{2}\right), i=1, \cdots, k$. Express the $Y_{i}$ 's using the linear regression model $\mathbf{Y}=\mathbf{X} \mu+\boldsymbol{\epsilon}$ where $\mathbf{Y}=\left(Y_{1}, \cdots, Y_{k}\right)^{\prime}, \mathbf{X}=\left(\sqrt{n_{1}}, \cdots, \sqrt{n_{k}}\right)^{\prime}$, 
and $\boldsymbol{\epsilon}=\left(\epsilon_{1}, \cdots, \epsilon_{k}\right)^{\prime}$ with the $\epsilon_{i}{ }^{\prime}$ 's being independently and identically distributed $N\left(0, \sigma^{2}\right)$ random variables. The results for linear regression give immediately the estimators

$$
\begin{aligned}
& \hat{\mu}=r\left(W_{01}+\cdots+W_{0 k}\right) \sim N\left(\mu, r \sigma^{2}\right) \text { with } r=\frac{1}{m_{1}+\cdots+m_{k}} \\
& \hat{\sigma}^{2}=\frac{1}{\nu} \sum_{j=1}^{k}\left(\frac{W_{0 j}-m_{j} \hat{\mu}}{\sqrt{m_{j}}}\right)^{2} \sim \sigma^{2} \chi_{\nu}^{2} / \nu \text { with } \nu=k-1
\end{aligned}
$$

and $\hat{\mu}$ and $\hat{\sigma}^{2}$ are independent.

Since the weight of a future bag $W_{i}$ and the calibration data $\mathcal{E}$ are independent,

$$
\frac{W_{i}-n_{i} \hat{\mu}}{\hat{\sigma} \sqrt{n_{i}+r n_{i}^{2}}}
$$

has a $t$ distribution with $\nu$ df. Following Neyman's (1937) method and the form of $\mathcal{C}\left(W_{i}\right)$ in (1), we construct the following confidence set for $n_{i}$ :

$$
\mathcal{C}\left(W_{i}\right)=\left\{n: \frac{W_{i}-n \hat{\mu}}{\hat{\sigma} \sqrt{n+r n^{2}}} \leq c\right\}, \quad i=1,2, \cdots
$$

where $c$ is a suitably chosen critical constant whose determination is considered next.

As in Section 2, it is desirable that the proportion of the future confidence sets $\mathcal{C}\left(W_{i}\right)(i=$ $1,2, \cdots)$ that include the true $n_{i}$ should be no less than the pre-specified $\beta$, that is,

$$
\liminf _{N \rightarrow \infty} \frac{1}{N} \sum_{i=1}^{N} I_{\left\{n_{i} \in C\left(W_{i}\right)\right\}} \geq \beta
$$

While the lower limit above depends on the $n_{i}$ 's in a complicated manner, it is shown in the appendix that this lower limit is bounded from below by $\inf _{n_{i} \in \mathcal{N}} E_{W_{i} \mid \mathcal{E}} I_{\left\{n_{i} \in C\left(W_{i}\right)\right\}}$, where $E_{W_{i} \mid \mathcal{E}}$ denotes the conditional expectation with respect to the random variable $W_{i}$ conditioning on the calibration data $\mathcal{E}$ (or, equivalently, $\hat{\mu}$ and $\hat{\sigma}$ ), and $\mathcal{N}$ denotes a set of natural numbers that contains all the future $n_{i}$ values. Although the future $n_{i}$ values are unknown, it is sensible to assume that all the future $n_{i}$ values are in a known range $\left[n_{l}, n_{u}\right]$. For example, all future $n_{i}$ 's must be at least $n_{l}, n_{l}=10$ say, since one can easily count the exact number $n_{i}$ of coins in a bag if $n_{i}$ is smaller than $n_{l}$. Similarly, one can easily set a conservative upper 
limit $n_{u}$ from the capacity of the weighing scale. Hence it is assumed in the rest of this paper that all the future $n_{i}$ values are contained in the known $\mathcal{N}=\left\{n: n_{l} \leq n \leq n_{u}\right\}$.

It follows therefore that a sufficient condition for guaranteeing (5) is

$$
\inf _{n_{i} \in \mathcal{N}} E_{W_{i} \mid \mathcal{E}} I_{\left\{n_{i} \in C\left(W_{i}\right)\right\}} \geq \beta
$$

Now, from the definition of $\mathcal{C}\left(W_{i}\right)$ in (4), a few lines of manipulation show that

$$
\inf _{n_{i} \in \mathcal{N}} E_{W_{i} \mid \mathcal{E}} I_{\left\{n_{i} \in C\left(W_{i}\right)\right\}}=\inf _{n_{i} \in \mathcal{N}} \Phi\left(\sqrt{r n_{i}} Z+c \sqrt{1+r n_{i}} X\right)
$$

where $Z=(\hat{\mu}-\mu) / \sqrt{r \sigma^{2}} \sim N(0,1), X=\hat{\sigma} / \sigma \sim \sqrt{\chi_{\nu}^{2} / \nu}$, and $Z$ and $X$ are independent random variables. Since the last expression in (7) depends on the random variables $\hat{\mu}$ and $\hat{\sigma}$ (via $Z$ and $X$ ), the condition in (6) cannot be guaranteed for all $\hat{\mu}$ and $\hat{\sigma}$. For example, if the values of $\hat{\mu}$ and $\hat{\sigma}$ are such that both $Z$ and $X$ are close to zero then $\Phi\left(\sqrt{r n_{i}} Z+c \sqrt{1+r n_{i}} X\right)$ is close to $1 / 2$ and hence smaller than $\beta \in(1 / 2,1)$ for each $n_{i} \in \mathcal{N}$. We therefore guarantee (6) with a large probability $1-\alpha$ with respect to the randomness in $\hat{\mu}$ and $\hat{\sigma}$ :

$$
P_{\mathcal{E}}\left\{\inf _{n_{i} \in \mathcal{N}} \Phi\left(\sqrt{r n_{i}} Z+c \sqrt{1+r n_{i}} X\right) \geq \beta\right\} \geq 1-\alpha,
$$

which in turn guarantees that

$$
P_{\mathcal{E}}\left\{\liminf _{N \rightarrow \infty} \frac{1}{N} \sum_{i=1}^{N} I_{\left\{n_{i} \in C\left(W_{i}\right)\right\}} \geq \beta\right\} \geq 1-\alpha
$$

The interpretation of this statement is as follows. Based on one set of calibration data $\mathcal{E}$ only, one constructs confidence sets $\mathcal{C}\left(W_{i}\right)$ for infinitely many future $n_{i}$ 's $(i=1,2, \cdots)$ and claims that at least ' $\beta$ proportion' of these confidence sets do contain the true $n_{i}$ 's. We repeat this process for a large number of times, say $\mathrm{R}$. Then, for $(1-\alpha) R$ of the times, the claim about ' $\beta$ proportion' is correct.

If one is interested in only one future $n_{i}$ then the critical constant $c$ in (4) can be set as $c=t_{\nu, \beta}$, the $\beta$-quantile of the $t_{\nu}$ distribution, and the resultant confidence set $C\left(W_{i}\right)$ in (4) is 
a usual $\beta$ level confidence set for one $n_{i}$ with the following coverage frequency interpretation: carry out one calibration experiment to collect $\mathcal{E}$ and measure the weight $W_{i}$ of one future bag, based on which to compute the confidence set $C\left(W_{i}\right)$ for this one $n_{i}$; then the frequency of the confidence sets that contain the corresponding $n_{i}$ 's is approximately $\beta$ among a large number of confidence sets constructed in this way. Note that, in this construction method, one calibration data set $\mathcal{E}$ is used only once with one future $W_{i}$ to produce one confidence set, and so the randomness in one $\mathcal{E}$ and the randomness in one future $W_{i}$ are treated on equal footing. This is clearly different from what is considered in this section and of interest to the company: the data $\mathcal{E}$ from one calibration experiment only is used repeatedly for inferences for infinitely many future $n_{i}$ values. Hence our proposed new method treats the two sources of randomness in $\mathcal{E}$ and a future $W_{i}$ differently.

We now consider how to compute the critical constant $c$ so that the probability in (8) is equal to $1-\alpha$. Note that this probability is equal to $P_{\mathcal{E}}\{(Z, X) \in \Omega\}$ where

$$
\Omega=\left\{\sqrt{r n_{i}} Z+c \sqrt{1+r n_{i}} X \geq z_{\beta} \text { for all } n_{i} \in \mathcal{N}\right\}=\cap_{n_{l} \leq n_{i} \leq n_{u}} \Omega_{n_{i}}
$$

with $\Omega_{n_{i}}=\left\{\sqrt{r n_{i}} Z+c \sqrt{1+r n_{i}} X \geq z_{\beta}\right\}$. Note that $\Omega_{n_{i}}$ is given by all the points, in the $(Z, X)$-half-plane with $X>0$, that are above the straight line $\sqrt{r n_{i}} Z+c \sqrt{1+r n_{i}} X=z_{\beta}$. Hence $\Omega$ is a region in the $(Z, X)$-half-plane with $X>0$ that is bounded by $n_{u}-n_{l}+1$ straight line segments. It follows directly that, for a given $c>0, P_{\mathcal{E}}\{(Z, X) \in \Omega\}$ can be computed easily using only one-dimensional numerical quadrature. Note further that $P_{\mathcal{E}}\{(Z, X) \in \Omega\}$ is strictly increasing in $c>0$. Hence, for a given $1-\alpha$, a routine numerical searching algorithm can be used to find the $c$ so that $P_{\mathcal{E}}\{(Z, X) \in \Omega\}=1-\alpha$. The Matlab programs for computing this $c$ and the lower confidence bounds are available from the journal website as supplementary material.

It is straightforward to show that the confidence set $\mathcal{C}\left(W_{i}\right)$ in (4) is given by the lower 
confidence bound

$$
L\left(W_{i}\right)= \begin{cases}\frac{2 \hat{\mu} W_{i}+c^{2} \hat{\sigma}^{2}-c \hat{\sigma} \sqrt{c^{2} \hat{\sigma}^{2}+4 \hat{\mu} W_{i}+4 r W_{i}^{2}}}{2\left(\hat{\mu}^{2}-r c^{2} \hat{\sigma}^{2}\right)} & \text { if } \hat{\mu}^{2}-r c^{2} \hat{\sigma}^{2} \neq 0 \\ \frac{W_{i}^{2}}{2 \hat{\mu} W_{i}+c^{2} \hat{\sigma}^{2}} & \text { otherwise }\end{cases}
$$

and that the distance between the lower confidence bound $L\left(W_{i}\right)$ and point estimator $W_{i} / \hat{\mu}$ of $n_{i}$ is increasing in $\hat{\sigma}, \beta$ and $1-\alpha$ as expected. Finally, taking into consideration the priori information that $n_{l} \leq n_{i} \leq n_{u}$, the lower confidence bound should be

$$
\bar{L}\left(W_{i}\right)=\min \left(n_{u}, \max \left(n_{l}, L\left(W_{i}\right)\right)\right)
$$

\section{An example}

In the calibration experiment, eleven bags of one-penny coins of known numbers $\left(m_{1}, \cdots, m_{11}\right)$ $=(100,200, \cdots, 1100)$ have been weighed to give the corresponding weights (grams) $\left(W_{0,1}, \cdots\right.$, $\left.W_{0,11}\right)=(356.97,716.67,1060.96,1427.10,1781.28,2130.23,2489.94,2849.74,3223.32,3575.76$, 3907.94). The data give $\hat{\mu}=3.564$ with $r=1 / 6600$ and $\hat{\sigma}^{2}=0.101$ with degrees of freedom $\nu=10$.

Let us assume that the number of coins in each future bags $n_{i}$ is known a priori to be between $n_{l}=100$ and $n_{u}=1400$, and set $\beta=0.95$ and $\alpha=0.05$. Then the critical constant in (4) is computed by our program to be $c=2.833$. Now for any future bag with weight $W_{i}$, one can use the formula in (11) to compute the lower confidence bound $\bar{L}\left(W_{i}\right)$ on $n_{i}$. For example, if $W_{i}=4000$ then $\bar{L}\left(W_{i}\right)=1113.3$ while the point estimate is 1122.5 ; if $W_{i}=4600$ then $\bar{L}\left(W_{i}\right)=1280.9$ while the point estimate is 1290.8 . These results show that the lower confidence bounds are pretty close to the point estimates. The advantage of the lower confidence bounds over the point estimates is of course the confidence statement (9) associated with the lower confidence bounds. 
If we treat 3.564 and 0.101 as the known values of $\mu$ and $\sigma^{2}$, respectively, then the formula in (3) can be used to compute the lower confidence bound $L\left(W_{i}\right)$ on $n_{i}$, which has nothing to do with $\alpha$. For example, if $W_{i}=4000$ then $L\left(W_{i}\right)=1117.5$ while the point estimate is still 1122.5; if $W_{i}=4600$ then $L\left(W_{i}\right)=1285.6$ while the point estimate is still 1290.8 . The differences between the two lower confidence bounds in (3) and (11) are therefore small at least in this example.

To get some idea on how sensitive the critical constant $c$ and the lower confidence bound $\bar{L}\left(W_{i}\right)$ in $(11)$ are to the bounds $n_{l}$ and $n_{u}$, we have computed $c, \bar{L}(400)$ and $\bar{L}(4000)$ for various $\left(n_{l}, n_{u}\right)$. From the results given in Table 1 , one can see that, while $c$ does change with $\left(n_{l}, n_{u}\right)$, the lower confidence bounds $\bar{L}(400)$ and $\bar{L}(4000)$ are not sensitive to the small changes in $\left(n_{l}, n_{u}\right)$. Hence one may prefer to use more conservative bounds $\left(n_{l}, n_{u}\right)$, for example $\left(n_{l}, n_{u}\right)=(50,1500)$ instead of $\left(n_{l}, n_{u}\right)=(100,1400)$, to make sure that all the future $n_{i}$ are in the interval $\left[n_{l}, n_{u}\right]$.

Table 1: Critical constant $c, \bar{L}(4000)$ and $\bar{L}(400)$ for various $\left(n_{l}, n_{u}\right)$

\begin{tabular}{lrrrrrr}
\hline$\left(n_{l}, n_{u}\right)$ & $(100,1400)$ & $(50,1400)$ & $(150,1400)$ & $(100,1300)$ & $(100,1500)$ & $(50,1500)$ \\
\hline$c$ & 2.833 & 2.846 & 2.824 & 2.827 & 2.840 & 2.852 \\
$\bar{L}(4000)$ & 1113.33 & 1113.29 & 1113.36 & 1113.35 & 1113.31 & 1113.27 \\
$\bar{L}(400)$ & 109.58 & 109.56 & 109.58 & 109.58 & 109.57 & 109.56 \\
\hline
\end{tabular}




\section{Conclusions}

This paper considers how to construct lower confidence bounds on infinitely many future $n_{i}$ 's based on the data $\mathcal{E}$ collected from only one calibration experiment. The two sources of randomness, those in the calibration data $\mathcal{E}$ and in a future weight $W_{i}$, have therefore to be treated differently. The proposed construction method guarantees, with confidence level $1-\alpha$ about the randomness in $\mathcal{E}$, that the proportion of the lower confidence bounds that bound the true $n_{i}$ 's, based on one calibration data set $\mathcal{E}$ only, is at least $\beta$, where $\alpha \in(0,0.5)$ and $\beta \in(0.5,1)$ are pre-specified.

If the two sources of randomness are treated on equal footing, then a standard lower confidence bound on one $n_{i}$, having the usual coverage frequency $\beta$, can be constructed by using (4) with $c=t_{\nu, \beta}$. But this is not of interest due to the repeated use of the same $\mathcal{E}$ in the construction of lower confidence bounds for infinitely many future $n_{i}$ 's.

While the interpretation of the proposed lower confidence bounds is somewhat more involved than a standard lower confidence bound, they have the same advantage as a standard lower confidence bound in terms of providing plausible bounds on the $n_{i}$ 's.

It is clear from Section 3 that the accuracy of $\hat{\sigma}^{2}$ depends only on the number $k$ of observed bags in the calibration experiment, and the total number of coins $m_{1}+\cdots+m_{k}$ in the $k$ bags only affects the accuracy of $\hat{\mu}$. It is interesting to study the optimal design of the calibration experiment which not only produces good lower confidence bounds in a certain sense but also

reduces the cost and possible human errors in counting the coins manually. The calibration experiment given in the example in Section 4 is simply based on the intuition that the $m_{j}$ 's should have a good spread over the possible range of the future $n_{i}$ values.

While the available information indicates that it is reasonable to assume the weight of a 
one-penny coin has a normal distribution, it is interesting to assess the impact on the lower confidence bounds developed in this paper and to develop suitable methods if the distribution is not normal. It seems that non-normality is likely to impact the optimal design of the calibration experiment too.

Finally, the key structure of the counting by weighing problem considered in this paper is that the statistical model estimated from one calibration date set is used repeatedly for inference related to infinitely many future observations and/or parameters. Our approach of treating differently the two sources of randomness involved have the potential to deal with other statistical problems that have a similar structure. For example, the statistical classification of all future cases is usually based on only one training data set. Given that the classification of a future case can be dealt with by constructing a confidence set on the true class of the case, our approach can be used to develop a new classification procedure that guarantees the frequency of correct classification of future cases with a certain confidence level about the randomness in the training data set. This problem is currently under investigation.

Acknowledgements: We sincerely thank the AE and referees for many constructive comments. Wei Liu would like to thank Professor Dave Nickerson for introducing counting by weighing to him.

\section{Appendix: Mathematical details}

We show that

$$
\inf _{n_{i} \in \mathcal{N}} E_{W_{i} \mid \mathcal{E}} I_{\left\{n_{i} \in C\left(W_{i}\right)\right\}} \leq \liminf _{N \rightarrow \infty} \frac{1}{N} \sum_{i=1}^{N} I_{\left\{n_{i} \in C\left(W_{i}\right)\right\}}
$$

where $E_{W_{i} \mid \mathcal{E}}$ denotes the conditional expectation with respect to the random variable $W_{i}$ conditioning on the calibration data $\mathcal{E}$ (or, equivalently, $\hat{\mu}$ and $\hat{\sigma}$ ) and $\mathcal{N}$ denotes the set of 
natural numbers known a priori to contain all the future $n_{i}(i=1,2, \cdots)$ values.

Note from the classical strong law of large numbers (cf. Chow and Teicher, 1978) that

$$
\lim _{N \rightarrow \infty} \frac{1}{N} \sum_{i=1}^{N}\left[I_{\left\{n_{i} \in C\left(W_{i}\right)\right\}}-E_{W_{i} \mid \mathcal{E}} I_{\left\{n_{i} \in C\left(W_{i}\right)\right\}}\right]=0
$$

and so

$$
\begin{aligned}
& \liminf _{N \rightarrow \infty} \frac{1}{N} \sum_{i=1}^{N} I_{\left\{n_{i} \in C\left(W_{i}\right)\right\}} \\
= & \lim _{N \rightarrow \infty} \frac{1}{N} \sum_{i=1}^{N}\left[I_{\left\{n_{i} \in C\left(W_{i}\right)\right\}}-E_{W_{i} \mid \mathcal{E}} I_{\left\{n_{i} \in C\left(W_{i}\right)\right\}}\right]+\liminf _{N \rightarrow \infty} \frac{1}{N} \sum_{i=1}^{N} E_{W_{i} \mid \mathcal{E}} I_{\left\{n_{i} \in C\left(W_{i}\right)\right\}} \\
= & \liminf _{N \rightarrow \infty} \frac{1}{N} \sum_{i=1}^{N} E_{W_{i} \mid \mathcal{E}} I_{\left\{n_{i} \in C\left(W_{i}\right)\right\}} .
\end{aligned}
$$

The required result in (12) now follows immediately from

$$
\frac{1}{N} \sum_{i=1}^{N} E_{W_{i} \mid \mathcal{E}} I_{\left\{n_{i} \in C\left(W_{i}\right)\right\}} \geq \inf _{n_{i} \in \mathcal{N}} E_{W_{i} \mid \mathcal{E}} I_{\left\{n_{i} \in C\left(W_{i}\right)\right\}}
$$

for any $N \geq 1$ since it is known that all the $n_{i} \in \mathcal{N}$.

\section{References}

Arntzen, F.K., Mulder, J.G. and Visser, J.H.M. (1994). Rapid and nondestructive assessment of the number of eggs in cysts of potato cyst nematodes by weighing. Fundamental and Applied Nematology, 17, 299-301.

Chow, Y.S. and Teicher, H. (1978). Probability Theory: Independence, Interchangeability, Martingales. Springer-Verlag, New York.

Guttman, I. and Menzefricke, U. (1986). Counting by weighing: an approach using renewal theory. Journal of the American Statistical Association, 81, 129-131.

Mullennix, P. (1990). Accurate estimation of count totals using ratio estimators. Journal of Quality Technology, 22, 277-282. 
Nelson, P.R. (1983). Counting a large number of items using the weight of a small sample. Technometrics, 25, 173-177.

Nickerson, D.M. (1993). Another look at counting by weighing. Commun. Statist. - Simula., 22, 323-343.

Nickerson, D.M. (2003). Addendum to counting by weighing and the seed testing problem. Annals of Applied Biology, 143, 371-374.

Nickerson, D.M. (2008). Counting by weighing and its effect on comparing population proportions Statistics \& Probability Letters, 78, 2321-2326.

Neyman, J. (1937). Outline of a theory of statistical estimation based on the classical theory of probability. Philosophical Transaction of the Royal Society of London, Series A, Mathematical and Physical Sciences, 236, 333-380.

Ridout, M.S. and Roberts, S.J. (1997). Improving quality control procedures for seed-borne pathogens by testing several samples of seeds. Seed Science and Technology, 25, 195-202.

Ridout, M.S., Suntheralingam, N. (1997). Counting by weighing and its effect on seed testing procedures. Annals of Applied Biology, 130, 179-185.

Yu, K.F. (1989). On a problem of counting by weighing. The Canadian Journal of Statistics, $17,377-389$. 